\title{
ARTí́CULO \\ Desarrollo osteológico de la columna vertebral y del complejo caudal de larvas del pez payaso Amphiprion ocellaris (Pomacentridae) en condiciones de cultivo
}

Osteological development of the vertebral column and caudal complex of the clownfish Amphiprion ocellaris (Pomacentridae) larvae under rearing conditions

\section{Estela Rodríguez-Ibarra ${ }^{1, *}$, María Isabel Abdo-de la Parra ${ }^{1}$, Gabriela Velasco-Blanco ${ }^{1}$ y Gabriela Aguilar-Zárate ${ }^{1}$} ${ }^{1}$ Centro de Investigación en Alimentación y Desarrollo, A.C., Av. Sábalo Cerritos, S.N. C.P. 82100 Mazatlán, Sinaloa, México.
*eibarra@ciad.mx

\begin{abstract}
The osteological development of the vertebral column and the caudal complex of the clownfish Amphiprion ocellaris, an important ornamental species is described. The organisms were reared under controlled conditions from hatching until 30 days post hatch (DPH) and methods for the clearing and differential staining of bone and cartilage tissues were applied. At hatching time, most of the vertebral elements had developed in cartilage stage and the notochord in pre-flexion larvae; 11 neural arches were located in the abdominal region while 12 neural arches and 12 haemal arches were found in the caudal region. In the caudal complex, the hypurals were still separated from one another. On $6 \mathrm{DPH}$ the notochord was in flexion stage and the ventral ribs were observed, while 12 neural spines and 12 haemal spines had developed in the caudal region and the hypurals were fused. The notochord was in the post-flexion stage on $11 \mathrm{DPH}$ and the complex caudal completely formed with all the neural spines and haemal spines fully developed. At $23 \mathrm{DPH}$ all structures had ossified. The vertebral column was divided in the abdominal region and comprised of 11 vertebrae while in the caudal region there were 15 vertebrae including the urostyle. Detailed information of osteological structures serve as a reference for the timely detection of malformations during clownfish aquaculture.
\end{abstract}

Key words: Amphiprion ocellaris, ornamental fish, larviculture, clearing and staining, osteology

Resumen.- Se describe el desarrollo osteológico de la columna vertebral y del complejo caudal de Amphiprion ocellaris, una especie de importancia ornamental. Los organismos se cultivaron bajo condiciones controladas desde la eclosión hasta los 30 días después de eclosionar (DDE) y fueron procesados con los métodos de clareado y tinción diferencial de tejidos óseos y cartilaginosos. Las larvas al eclosionar ya tienen desarrollados la mayoría de sus elementos vertebrales en etapa de cartílago y el notocordio en estado de pre-flexión; la región abdominal presenta 11 arcos neurales y en la región caudal 12 arcos neurales y 12 arcos hemales. En el complejo caudal se observaron los hipurales aún separados entre ellos. A los 6 DDE el notocordio se encontró en etapa de flexión y aparecen las costillas ventrales, y en la región caudal se desarrollaron 12 espinas neurales y 12 espinas hemales y los hipurales ya se han fusionados. A los 11 DDE el notocordio ya estaba en pos-flexión y el complejo caudal completamente formado, y se desarrollaron completamente todas las espinas neurales y espinas hemales. A los 23 DDE se observó la osificación de todas las estructuras. La columna vertebral de A. ocellaris se divide en una región abdominal, con 11 vértebras y una región caudal con 15 vértebras incluyendo el urostilo. El conocimiento detallado de las estructuras osteológicas servirá de referencia para la detección oportuna de malformaciones durante el cultivo del pez payaso.

Palabras clave: Amphiprion ocellaris, peces ornamentales, larvicultura, aclaración y tinción diferencial, osteología

\section{INTRODUCCIÓN}

En la actualidad la acuariofilia de peces marinos ha ido en expansión a nivel mundial y México no es la excepción, convirtiéndose en un pasatiempo popular y cada vez se intensifica el comercio de peces de ornato. Entre las especies más solicitadas y mejor cotizadas por los acuaristas está el pez payaso Amphiprion ocellaris (Cuvier, 1830), que pertenece a la familia Pomacentridae y quien agrupa a otras especies de ornato que son aproximadamente el $50 \%$ de las especies comercializadas, siendo el pez payaso una de las 10 especies de mayor demanda (Lango-Reynoso et al. 2012). Actualmente, esta especie se está cultivando en las instalaciones de la Unidad Mazatlán del Centro de Investigación en Alimentación y Desarrollo (CIAD), obteniendo cosechas constantes de juveniles, los cuales son comercializados y al mismo tiempo se 
están realizando diversas investigaciones para mejorar su cultivo (Abdo de la Parra et al. 2013, Velasco-Blanco et al. 2016).

Uno de los problemas graves que se presenta en los criaderos de peces es la presencia de malformaciones esqueléticas (lordosis, cifosis, escoliosis, fusión de vertebras, etc.) por diversos factores ya sea genéticos, nutricionales, condiciones de cultivo, entre otros (Fraser et al. 2004, Hernández et al. 2013), y el pez payaso no es la excepción, ya que durante cada ciclo de cultivo se ha dado la presencia de peces deformes, lo cual incide negativamente en la producción de los organismos y se ve reflejado en pérdidas económicas (Boglioni et al. 2001, Fernández et al. 2008). Si bien, se ha observado que dichas deformidades no les afecta aparentemente en sus funciones básicas, la finalidad de su reproducción y crianza en cautiverio es la obtención de organismos con características externas normales y por consiguiente saludables.

El desarrollo osteológico en larvas de peces es un proceso detallado que se inicia con la formación de cartílago y su posterior osificación. Tal es el caso del complejo caudal, donde sus elementos que lo conforman en algún momento se fusionan o desaparecen durante el proceso. El conocimiento de la estructura ósea del pez payaso y su evaluación temprana de las características merísticas como con el número de vértebras y/o componentes esqueléticos será útil para detectar oportunamente alguna deformación y descartar de la producción aquellos peces que la presenten. Por lo tanto, el objetivo del presente trabajo fue describir los elementos que conforman la columna vertebral y del complejo caudal de las larvas del pez payaso, para generar una herramienta de referencia y poder evaluar la calidad durante su cultivo.

\section{Materiales Y MÉTODOS}

Las larvas del pez payaso fueron obtenidas de un desove de una pareja de reproductores en cautiverio, pertenecientes al CIAD unidad Mazatlán. El cultivo larvario se llevó a cabo en tanques de $300 \mathrm{~L}$ bajo condiciones óptimas, con una temperatura de $28^{\circ} \mathrm{C}$, salinidad de 33 y $6,2 \mathrm{mg} \mathrm{L}^{-1}$ de oxígeno. Se usó la técnica de agua verde y del día 1 al 9 después de la eclosión (DDE) se añadieron microalgas Nannochloropsis oculata e Isochrysis galbana, asimismo se agregaron rotíferos de la especie Brachionus rotundiformis (10-20 organismos $\mathrm{mL}^{-1}$ ). Para el $9 \mathrm{DDE}$ se añadió al tanque de cultivo nauplios de Artemia sp. y posteriormente adultos enriquecidos. A partir del 19 DDE se empezó a destetar a los organismos mediante alimento comercial para peces de ornatos en forma de hojuela (Abdo- de la Parra et al. 2013).

Se tomaron muestras de peces desde el primer día de nacidos hasta el día 30 , los cuales primeramente se sacrificaron con el mínimo de estrés mediante una sobredosis del anestésico 2-fenoxietanol (Sigma Aldrich). Se registró la longitud total (LT) de los especímenes, utilizando un vernier digital con una resolución de $0,01 \pm 0,03 \mathrm{~mm}$, y posteriormente se procesaron con la técnica de diafanización (clareado) y tinción diferencial de hueso y cartílago (doble tinción) descrita por Potthoff (1984) con algunas modificaciones.

Los especímenes fueron fijados por $24 \mathrm{~h}$ en formol (Hycel) al $10 \%$ en buffer fosfato. La deshidratación se realizó por $24 \mathrm{~h}$ en etanol al $50 \%$ (Fisher) y posteriormente a etanol $100 \%$ otras 24 h. La tinción de cartílago de los especímenes se llevó a cabo de 24 a $48 \mathrm{~h}$ (de acuerdo al tamaño) utilizando $70 \mathrm{ml}$ etanol absoluto, $30 \mathrm{ml}$ ácido acético (JT Baker) y $20 \mathrm{mg}$ de azul aciano (Fluka). La neutralización fue de $12 \mathrm{~h}$ con borato de sodio $\left(\mathrm{Na}_{2} \mathrm{~B}_{4} \mathrm{O}_{7}\right)$ (Fisher) saturado. Para realizar el blanqueamiento de los especímenes se usó $15 \mathrm{ml}$ de peróxido $\left(\mathrm{H}_{2} \mathrm{O}_{2}\right)$ (Fermont) al 3\% y $85 \mathrm{ml}$ de hidróxido de potasio (KOH) (Fisher) al 1\% durante $40 \mathrm{~min}$. En especímenes pequeños se aplicó esta solución por unos cuantos segundos, hasta observar que no se formaran burbujas. El aclaramiento de los ejemplares se llevó a cabo utilizando $35 \mathrm{ml}$ de $\mathrm{Na}_{2} \mathrm{~B}_{4} \mathrm{O}_{7}$ saturado, $65 \mathrm{ml}$ agua destilada y $50 \mathrm{mg}$ de tripsina (Sigma Aldrich). Es importante señalar que, el tiempo de digestión de la enzima sobre el músculo de los especímenes varió de acuerdo al tamaño del mismo.

La tinción de hueso se realizó utilizando $100 \mathrm{ml} \mathrm{KOH} \mathrm{y} 10$ mg rojo alizarina (Sigma Aldrich) con una duración de 1 a 4 días de acuerdo al tamaño del espécimen. Para realizar la transparentación, se usó $35 \mathrm{ml} \mathrm{Na} \mathrm{B}_{4} \mathrm{O}_{7}$ saturado, $65 \mathrm{ml}$ agua destilada y $50 \mathrm{mg}$ de tripsina de 24 a 48 h, o bien hasta que se eliminaron los restos de color y que el músculo se terminara de digerir completamente. La decoloración se realizó utilizando $\mathrm{KOH}$ al $1 \%$ y el tiempo que le llevó al espécimen fue directamente proporcional al tamaño del mismo. Se hizo recambios de solución cada 10 días en los casos requeridos. Para la preservación de los especímenes se utilizó glicerina en $\mathrm{KOH}$ al $1 \%$ a diferentes concentraciones, primero al $30 \%$, después al $60 \%$ y finalmente al $100 \%$.

Cuando concluyó el proceso de aclarado y tinción de hueso y cartílago de los especímenes del pez payaso, se llevó a cabo el análisis de los mismos, el cual consistió en observar a través de un microscopio estereoscópico (Olympus ${ }^{\circledR} \mathrm{SZ}$ ) equipado con una cámara (Olympus ${ }^{\circledR}$ SP-350) con la que se tomaron fotos de la columna vertebral y del complejo caudal de los especímenes, en cada uno de los estadios de desarrollo, para hacer la descripción de estructuras óseas y cartilaginosas presentes. Los elementos osteológicos se describieron de acuerdo a la terminología utilizada por Lagler et al. (1990), 
Koumoundouros et al. (1997, 1999), Gavaia et al. (2002), Gállego (2008), Laggis et al. (2010) y Rodríguez-Ibarra et al . (2015).

\section{Resultados}

Las estructuras que componen la columna vertebral y el complejo caudal del pez payaso Amphiprion ocellaris se presentan en la Tabla 1, con sus respectivas abreviaciones utilizadas.

\section{Desarrollo de las estructuras de la columa VERTEBRAL Y DEL COMPLEJO CAUDAL DURANTE EL CULTIVO LARVARIO}

Las larvas recién eclosionadas midieron 4,0 mm LT, y se observó la presencia de la mayoría de sus elementos vertebrales, pero aún en etapa de cartílago. El notocordio se encontró en estado de pre-flexión, y en la región abdominal, se desarrollaron los 11 An y las estructuras cartilaginosas que darán lugar a las 6 P. Asimismo, en la región caudal ya se han formado los 12 An y los $12 \mathrm{Ah}$, en el complejo caudal se observó la formación de los $\mathrm{H}$ (separados entre ellos) y $\mathrm{Ph}$, así como la presencia de 2 de los 3 E, además de los 2 An y los 2 Ah (Fig. 1a).

Tabla 1. Elementos del esqueleto de Amphiprion ocellaris y sus abreviaciones / Skeletal elements of Amphiprion ocellaris clownfish and their abbreviations

\begin{tabular}{lc}
\hline \multicolumn{1}{c}{ Elemento del esqueleto } & Abreviatura \\
\hline Vértebras centrales & $\mathrm{Vc}$ \\
Arcos neurales & $\mathrm{An}$ \\
Espinas neurales & $\mathrm{En}$ \\
Costillas ventrales & $\mathrm{Cv}$ \\
Costillas dorsales & $\mathrm{Cd}$ \\
Parapófisis & $\mathrm{P}$ \\
Arco hemal & $\mathrm{Ah}$ \\
Espina hemal & $\mathrm{Eh}$ \\
Epurales & $\mathrm{E}$ \\
Hipurales & $\mathrm{H}$ \\
Parahipural & $\mathrm{Ph}$ \\
Espina neural modificada & $\mathrm{Enm}$ \\
Espina hemal modificada & $\mathrm{Ehm}$ \\
Arco neural especializado & $\mathrm{Ane}$ \\
Centros preurales & $\mathrm{Cp}$ \\
Urostilo & $\mathrm{U}$ \\
Uroneural & $\mathrm{Un}$ \\
Lepidotriquia & $\mathrm{L}$ \\
Dermatotriquia & $\mathrm{D}$ \\
Accesorio cartilaginoso & $\mathrm{Ac}$ \\
\hline
\end{tabular}

A los 6 DDE, se registró una LT promedio de las larvas de $9,2 \mathrm{~mm}$. El notocordio ya se encontró en etapa de flexión y en lo que se refiere a la región abdominal, aparecen las $\mathrm{Cv}$ y los 6 Py 3 En (de la 9 a la 11). En la región caudal, ya se han formado las 12 En y las 12 Eh; y en el complejo caudal los 3 E, el Ane, los Ac 1 y 2 así como la D y se observó la fusión de los $\mathrm{H}(1+2$ y $3+4)$. Cabe mencionar que todos estos elementos aún se encontraron en estado cartilaginoso (Fig. 1b).

A los 11 DDE (13,5 mm de LT), el notocordio se observó en estado de post-flexión y la placa hipúrica se encontró completamente formada. Se apreciaron ya desarrollados y osificados los P y todas las En y Eh con sus arcos respectivos. En la aleta caudal, también se han osificado la mayoría de los elementos a excepción de los E y del Ane. En el pedúnculo caudal se observó la aparición del Un también ya osificado y del Ac3 (Fig. 1c).

A los 23 DDE los especímenes alcanzaron una LT de 17,6 mm (Fig. 1d), se observó la osificación de todas las estructuras tanto de la región abdominal como caudal y la base de los radios caudales, solo hay presencia de cartílago en la base donde se insertan dichos radios y en la parte ventral de los Cp y del U. Las Vc han cambiado su cuerpo casi cuadrado y ya muestran su forma anficélica (cuerpo cilíndrico y extremos cóncavos) (Gállego 2008, Olivares \& Rojas 2013). A los 30 DDE que fue el día que culminó el experimento, los organismos tuvieron una LT promedio de 20,5 mm, se observó las Vc más alargadas, debido a que sus extremos son más cóncavos (Fig. 1e), así como la presencia de $\mathrm{Cd}$ osificadas (Fig. 2).

En el caso del complejo caudal, desde el 1 DDE se observó el desarrollo de varias de sus estructuras, con una clara separación entre los $\mathrm{H}$ y $\mathrm{Ph}$. Posteriormente, a los $6 \mathrm{DDE}$ ya se unieron los $\mathrm{H} 1$ y 2 así como el 3 y 4 para dar origen a 2 placas, y en el caso del $\mathrm{Ph}$ éste se encuentra unido solo de su base y fue hasta el 30 DDE que se observó fusionado casi en su totalidad a los $\mathrm{H} 1$ y 2 (Fig. 1).

\section{COLUMNA VERTEBRAL Y COMPLEJO CAUDAL}

En el presente estudio fueron procesados un total de 90 especímenes con un rango de tallas de 4,0 a 20,5 mm de longitud total (LT), los cuales fueron cultivados hasta el 30 DDE.

De acuerdo a Meunier \& Ramzu (2006), Gállego (2008), Bensimon-Brito et al. (2010), Olivares \& Rojas (2013) y Rodríguez-Ibarra et al. (2015), la columna vertebral (incluido el complejo caudal) se divide principalmente en dos regiones, la anterior o abdominal y la posterior o caudal, $11 \mathrm{Vc}$ son abdominales y 15 caudales incluyendo el U. Cada vértebra abdominal se compone por su parte dorsal de un An y un En, 


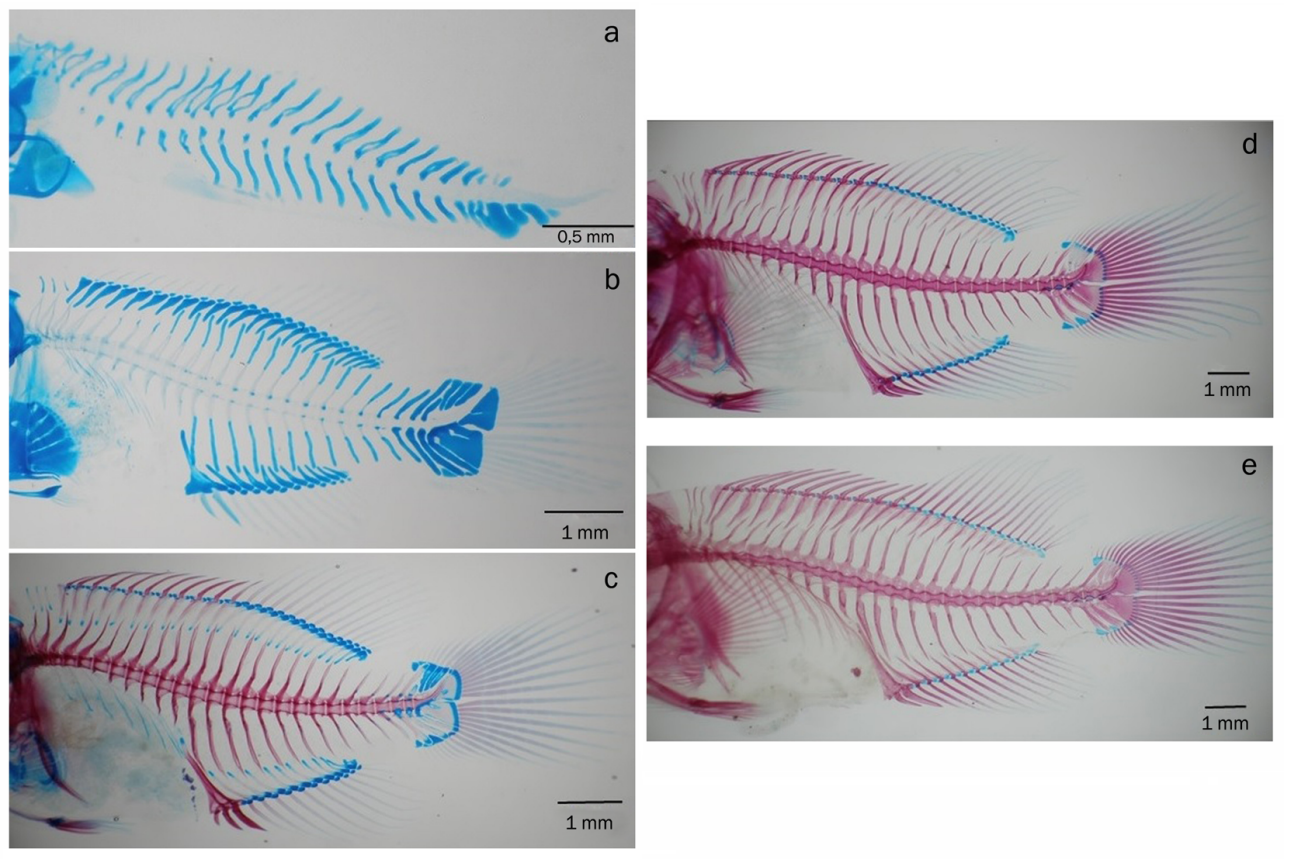

Figura 1. Desarrollo normal de la columna vertebral y complejo caudal del pez payaso Amphiprion ocellaris, en diferentes fases de desarrollo durante su cultivo. a) $3 \mathrm{~mm}$, b) $6 \mathrm{~mm}$, c) 8,2 mm, d) $13 \mathrm{~mm}$ y e) $17 \mathrm{~mm} /$ Normal development of the vertebral column and caudal complex of the Amphiprion ocellaris clownfish at different development stages during cultivation. a) $3 \mathrm{~mm}$, b) $6 \mathrm{~mm}$, c) $8.2 \mathrm{~mm}$, d) $13 \mathrm{~mm}$ and e) $17 \mathrm{~mm}$

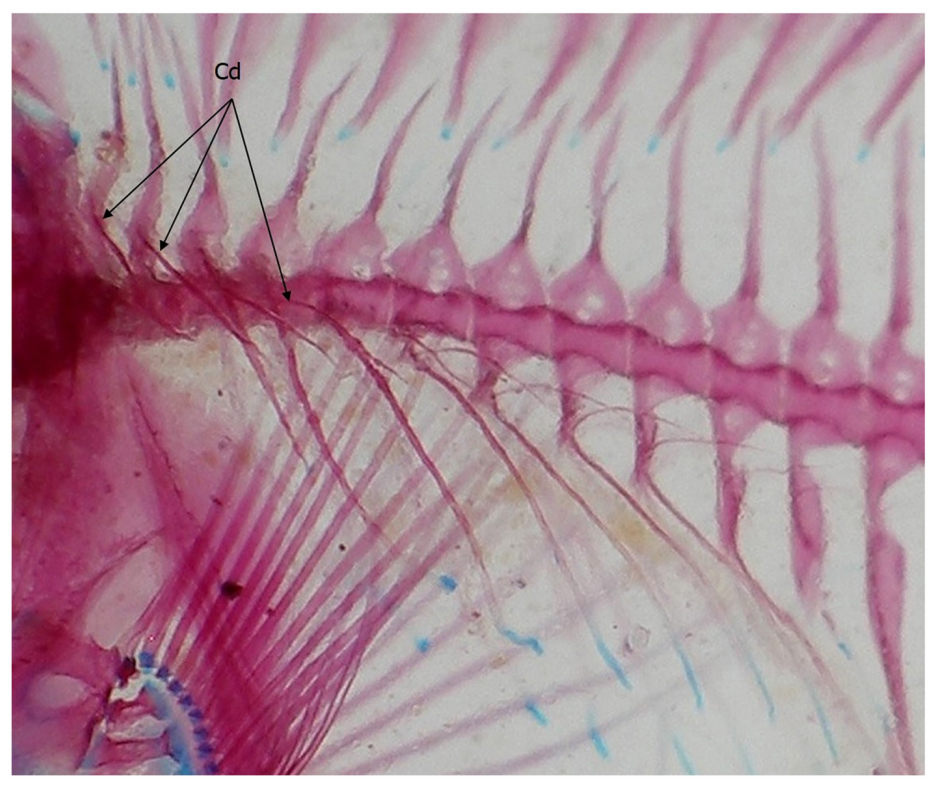

Figura 2. Costillas dorsales de Amphiprion ocellaris / Dorsal ribs of Amphiprion ocellaris

116 Rodríguez-lbarra et al.

Osteología vertebral y caudal del pez payaso 
mientras que por la parte ventral 6 de sus 8 vértebras están equipadas con P (Fig. 3). Las vértebras de la región caudal están equipadas con An y En dorsalmente y por Ah y Eh ventralmente. La última (Enm) y dos hemales (Ehm1 y Ehm2), se prolongan para dar soporte a los radios de la aleta caudal (Fig. 4).

La región abdominal también incluye $9 \mathrm{Cv}, 6 \mathrm{P}$ y $14 \mathrm{Cd}$. Asimismo, el complejo caudal se compone de 2 Cp y 1 U, 3 E, $4 \mathrm{H}$ (que se fusionaron 1 y 2,3 y 4 ), $1 \mathrm{Ph}, 1 \mathrm{Enm}, 1$ Ane, 2 Ehm y 1 Un (Fig. 3 y 4 ).

La aleta caudal está compuesta por 19 radios principales también llamada lepidotriquia (L), y está constituida por 2 lóbulos, uno dorsal y otro ventral ambos bajo el notocordio.
Son 9 radios en la parte inferior y 10 en la superior, los cuales se encuentra distribuidos y apoyados de la siguiente manera en las estructuras del complejo caudal: En la parte inferior, el radio 1 se apoya en la Ehm2, el segundo radio entre la Ehm y $\mathrm{Ph}$, el tercero en el $\mathrm{Ph}$ y los radios 4 al 9 en los $\mathrm{H} 1$ y H2. Mientras que los radios de la parte superior (1 al 10) se distribuyeron en los H3 y H4. La aleta caudal también cuenta con radios accesorios o procurrentes que son llamados dermatotriquia (D) y se encuentran distribuidos en los extremos de los radios principales. En la parte ventral estos radios se encuentran sobre la Ehm1 y sobre los Ac1 y Ac2 (Koumoundouros et al. 1997, 1999, 2001b; Laggis et al. 2010). En cuanto a los radios de la parte dorsal se encuentran distribuidos en los E, en la Enm y en el Ac3 (Fig. 4).

Figura 3. Estructuras osteológicas que componen la columna vertebral y complejo caudal de Amphiprion ocellaris / Osteological structures that make up the vertebral column and caudal complex of Amphiprion ocellaris
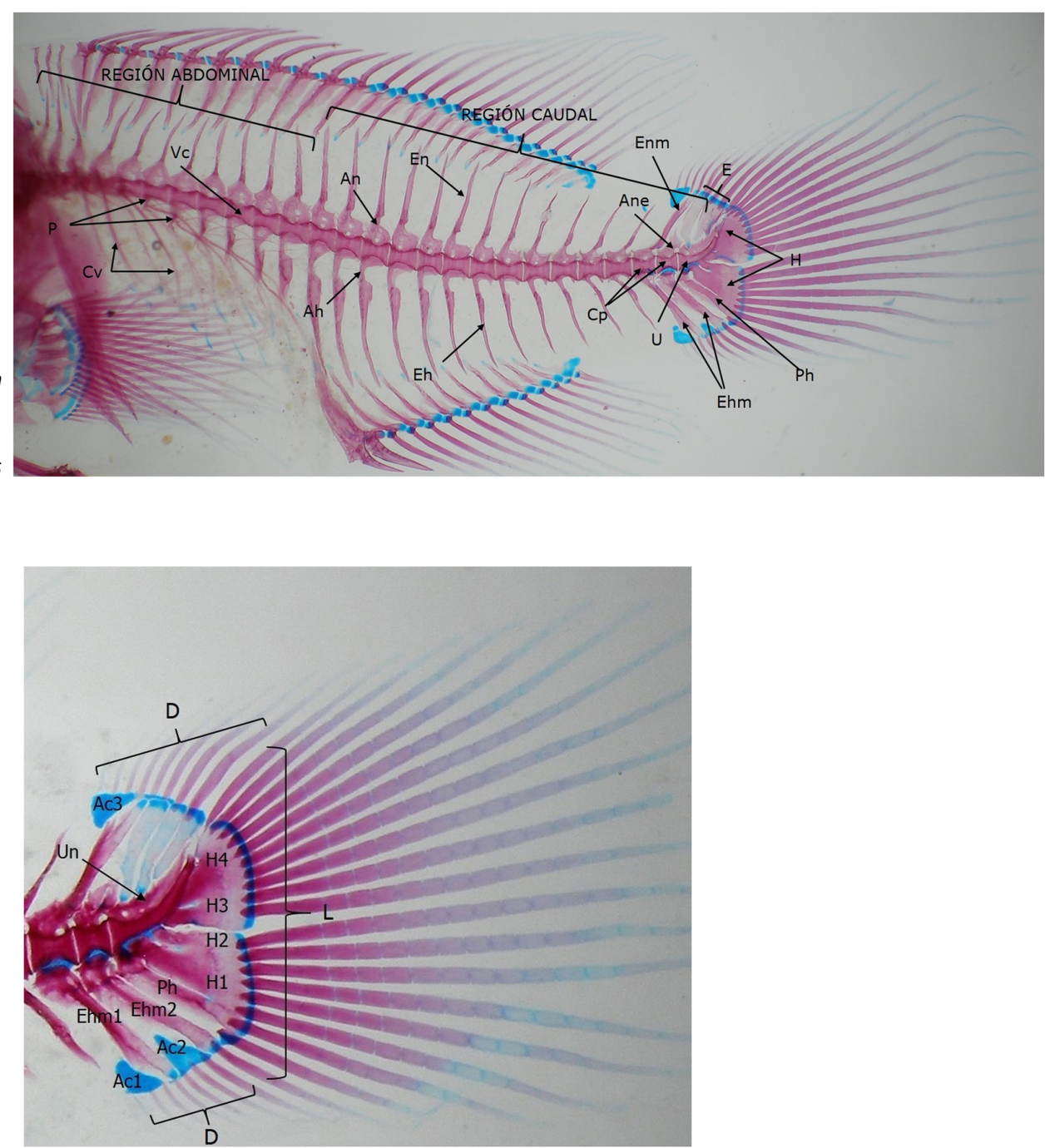

Figura 4. Elementos que componen el complejo caudal de Amphiprion ocellaris y distribución de sus radios / Development of the caudal complex and distribution of fin rays in Amphiprion ocellaris 


\section{Discusión}

En el presente estudio se describió el desarrollo de los componentes de la columna vertebral y complejo caudal del pez payaso A. ocellaris y su subsecuente osificación, donde se observó que las larvas recién eclosionadas ya presentan la mayoría de sus elementos esqueléticos, aunque en forma rudimentaria, al igual que $A$. melanopus (Green \& McCormick 2001). Esto contrasta con otras especies como Seriola dumerlii y Lutjanus guttatus (Laggis et al. 2010, RodríguezIbarra et al. 2015) y las especies de la familia Sparidae como Sparus aurata, Dentex dentex, Diplodus sargus (Koumoundouros et al. 1997, 1999 y 2001b), las cuales presentan un desarrollo más lento en comparación con el pez payaso. Esta variabilidad de estados de la osteología de los peces que se presentan cuando eclosionan, depende de la cantidad de vitelo que contenga cada una de las especies, donde algunos inmediatamente al eclosionar ya empiezan a desarrollar sus estructuras, mientras que otros lo hacen varios días después de la eclosión (Hernández et al. 2012).

El rápido desarrollo de las estructuras de la columna vertebral y del complejo caudal de larvas del pez payaso, no permitió observar y registrar con detalle la aparición de muchas de ellas como en otras especies. En el periodo comprendido del día de la eclosión de las larvas al 6 DDE, fue donde se manifestaron algunos cambios importantes, como la presencia de En y Eh; mientras que en el complejo caudal se presentó la fusión de los $\mathrm{H}$, la aparición de los E y el estado del notocordio de preflexión a flexión. Del 11 DDE en adelante se observó principalmente el progreso de la osificación.

De acuerdo al desarrollo osteológico que presentó el pez payaso el cual se describió hasta el 30 DDE en el presente estudio, se comparan ciertos sucesos que se presentaron durante este proceso con el trabajo de Green \& McCormick (2001), quienes hicieron una breve descripción osteológica de $A$. melanopus, pero en su estudio solo detallan hasta los 9 DDE. En el caso de las condiciones de cultivo fueron muy semejantes, ya que la temperatura fue de $28^{\circ} \mathrm{C}$ en ambos trabajos, siendo este parámetro de los que más influyen sobre el desarrollo de las estructuras de la columna vertebral y del complejo caudal (Çoban et al. 2009). Entre las diferencias de estas dos especies en cuanto al desarrollo de las estructuras se refiere, al eclosionar se encontró en estado de flexión al notocordio de $A$. melanopus, mientras que en $A$. ocellaris se presentó en preflexión, y fue hasta el 3 DDE que ya se observó en estado de flexión (Green \& McCormick 2001). En cuanto a la osificación de sus estructuras, en A. melanopus se muestran resultados donde ciertas estructuras se osificaron más rápidamente que en A. ocellaris. En el caso de la especie del presente estudio, es hasta el 11 DDE que se observó la osificación solo en lo que respecta a las Vc, An, En, Ah y Eh, no así en el complejo caudal donde quedaban estructuras por osificar; a diferencia de $A$. melanopus donde a partir del 6 DEE ya presentó una osificación completa de las estructuras de su esqueleto (Green \& McCormick 2001). Cabe mencionar la presencia en $A$. ocellaris de las Cd que se observaron a los $20,5 \mathrm{~mm}$ de LT (30 DDE) y que solo se hace mención de estas estructuras en el desarrollo osteológico de $D$. dentex las cuales se hicieron presentes a los $25 \mathrm{~mm}$ de LT (Koumoundouros et al. 1999).

En relación al complejo caudal o uróforo del pez payaso, que de acuerdo a Gállego (2008) es considerado como evolucionado, ya que presentó fusiones en sus hipurales (H1 y H2, H3 y H4) semejante a L. calcarifer (Fraser et al. 2004), S. dumerili (Laggis et al. 2010) y los espáridos S. aurata, D. dentex y D. sargus (Koumoundouros et al. 1997, 1999, 2001b); a diferencia de especies como el pez cebra y pargo (Bensimon-Brito et al. 2010, Rodríguez-Ibarra et al. 2015) que solo muestran fusión en las bases entre algunos $\mathrm{H}$ (Çoban et al. 2009). El desarrollo avanzado de las estructuras que componen el complejo caudal de A. ocellaris, podría representar una ventaja que tienen al eclosionar, ya que al tener esta zona del cuerpo desarrollada le otorga la fuerza locomotora necesaria para el nado y así obtener sin dificultades su alimento y por consiguiente aumentar su supervivencia, lo cual se ha observado en esta especie (Koumoundouros et al. 2001a, Hernández et al. 2016)

En el caso de Un, estructura que se manifestó en $A$. ocellaris a los 11 DDE y que de acuerdo a Laggis et al. (2010) su presencia es una característica de los teleósteos, y lo han reportado en especies como D. sargus y D. dentex (Koumoundouros et al. 1999, 2001b) y en carángidos como S. dumerili. Asimismo se desarrollaron 3 Ac, los cuales también se han observado en otras especies, y su número y distribución es característica de cada especie o taxón y puede ser una herramienta para la sistemática (Koumoundouros et al. 1997, 1999, 2001b; Laggis et al. 2010, Rodríguez-Ibarra et al. 2015).

El conocimiento del desarrollo normal osteológico del pez payaso A. ocellaris bajo condiciones de cultivo, contribuirá información básica para futuros trabajos en áreas como larvicultura y nutrición. En el caso del cultivo larvario, mejorar los protocolos y evaluar, entre otras cosas, parámetros ambientales que puedan incidir sobre el desarrollo de los organismos. En el caso nutricional, diseñar dietas tanto para reproductores como para larvas, además de enriquecer el alimento vivo; todo esto encaminado a mejorar la producción de organismos y disminuir las malformaciones esqueléticas que se puedan presentar durante los ciclos de cultivo. 


\section{Agradecimientos}

Los autores agradecen a V. Williams de Calvario por la traducción del resumen y al Sr. Juan Huerta por la asistencia técnica.

\section{LITERATURA CITADA}

Abdo-de la Parra MI, G Velasco-Blanco, LE Rodríguez-Ibarra \& N García-Aguilar. 2013. Cultivo larvario del pez payaso (Amphiprion ocellaris) en la planta piloto del CIAD, unidad Mazatlán. Industria Acuícola 7(4): 12-13. <https://issuu.com/ industriaacuicola/docs/industria_acuicola_vol._7.4_mayo/12>

Bensimon-Brito A, ML Cancela, A Huysseune \& PE Witten. 2010. The zebrafish (Danio rerio) caudal complex -a model to study vertebral body fusion. Journal of Applied Ichthyology 26: $235-238$

Boglione C, F Gagliardi, M Scardi \& S Cataudella. 2001. Skeletal descriptors and quality assessment in larvae and post-larvae of wild caught and hatchery-reared gilthead sea bream (Sparus aurata L. 1758). Aquaculture 192: 1-22.

Çoban D, C Suzer, HO Kamaci, S Saka \& K Firat. 2009. Early osteological development of the fins in the hatchery-reared red porgy, Pagrus pagrus (L. 1758). Journal of Applied Ichthyology 25: 26-32.

Fernández I, F Hontoria, JB Ortiz-Delgado, Y Kotzamanis, A Estévez, JL Zambonino-Infante \& E Gisbert. 2008. Larval performance and skeletal deformities in farmed gilthead sea bream (Sparus aurata) fed with graded levels of vitamin A enriched rotifers (Brachionus plicatilis). Aquaculture 283(1): 102-115.

Fraser MR, TA Anderson \& R de Nys. 2004. Ontogenic development of spine and spinal deformities in larval barramundi (Lates calcarifer) culture. Aquaculture 242: 697-711.

Gállego L. 2008. Los cordados. Funciones de control voluntario, 303 pp. Fundación Laboratorio de Biología Animal, Vigo.

Gavaia PJ, MT Dinis \& ML Cancela. 2002. Osteological development and abnormalities of vertebral column and caudal skeleton in larval and juvenile stages of hatchery-reared Senegal sole (Solea senegalensis). Aquaculture 211: 305-323.

Green BS \& MI McCormick. 2001. Ontogeny of digestive and feeding systems in the anemonefish Amphiprion melanopus. Enviromental Biology of Fishes 61: 73-83.

Hernández DR, JR Casciotta, JJ Santinón, S Sánchez \& HA Domitrovic. 2012. Desarrollo ontogénico de la columna vertebral y del esqueleto caudal en larvas de Rhamdia quelen en condiciones de larvicultura intensiva. International Journal of Morphology 30(4): 1520-1525.

Hernández DR, JJ Santinón, S Sánchez \& HA Domitrovic. 2013. Crecimiento, supervivencia e incidencia de malformaciones óseas en distintos biotipos de Rhamdia quelen durante la larvicultura. Latin American Journal of Aquatic Research 41(5): 877-887.
Koumoundouros G, F Gagliardi, P Divanach, C Boglione, S Cataudella \& M Kentouri. 1997. Normal and abnormal osteological development of caudal fin in Sparus aurata L. fry. Aquaculture 149: 215-226.

Koumoundouros G, P Divanach \& M Kentouri. 1999. Osteological development of the vertebral column and of the caudal complex in Dentex dentex. Journal of Fish Biology 54: 424-436.

Koumoundouros G, P Divanach \& M Kentouri. 2001a. Osteological development of Dentex dentex (Osteichthyes: Sparidae): dorsal, anal, paired fins and squamation. Marine Biology 138: 399-406.

Koumoundouros G, DG Sfakianakis, E Maingot, P Divanach \& M Kentouri. 2001b. Osteological development of the vertebral column and of the fins in Diplodus sargus (Teleostei: Perciformes: Sparidae). Marine Biology 139: 853-862.

Laggis A, DG Sfakianakis, P Divanach \& M Kentouri. 2010. Ontogeny of the body skeleton in Seriola dumerili (Risso, 1810). Italian Journal of Zoology 77(3): 303-315.

Lagler KF, JE Bardach, RR Miller \& DR May-Passino. 1990. Ictiología, 489 pp. AGT Editor, México.

Lango-Reynoso F, M Castañeda-Chávez, JE Zamora-Castro, G Hernández-Zárate, MA Ramírez-Barragán \& E SolísMorán. 2012. La acuariofilia de especies ornamentales marinas: Un mercado de retos y oportunidades. Latin American Journal of Aquatic Research 40(1): 12-21.

Meunier FJ \& MY Ramzu. 2006. La regionalization morphofonctionnelle de l'axe vertebral chez les Teléostéens en relation avec le mode de nage. Comptes Rendus Palevol 5: 499-507.

Olivares R \& M Rojas. 2013. Esqueleto axial y apendicular de vertebrado. International Journal of Morphology 31(2): 378387.

Potthoff T. 1984. Clearing and staining techniques. In: Moser HG, WJ Richards, DM Cohen, MP Fahay, AW Kendall Jr \& SL Richardson (eds). Ontogeny and systematics of fishes, Special Publication 1: 35-37. American Society of Ichthyologists and Herpetologists. Allen Press, Lawrence.

Rodríguez-Ibarra LE, MA Abdo-de la Parra, G Aguilar-Zárate, G Velasco-Blanco \& L Ibarra-Castro. 2015. Desarrollo osteológico de la columna vertebral y del complejo caudal de larvas de Lutjanus guttatus (Perciformes: Lutjanidae) en condiciones de cultivo. Revista Biología Tropical 63(1): 155164.

Velasco-Blanco G, MI Abdo-de la Parra \& LE RodríguezIbarra. 2016. Biotecnología para la producción de peces marinos de ornato en CIAD, Unidad Mazatlán. Panorama Acuícola 22(1): 30-32. <https://issuu.com/designpublications/ docs/panorama_acuicola_22-1> 